\title{
Influencing modulation properties of quantum-dot semiconductor lasers by carrier lifetime engineering
}

Cite as: Appl. Phys. Lett. 101, 131107 (2012); https://doi.org/10.1063/1.4754588

Submitted: 14 February 2012 - Accepted: 10 September 2012 • Published Online: 25 September 2012

Benjamin Lingnau, Kathy Lüdge, Weng W. Chow, et al.

ARTICLES YOU MAY BE INTERESTED IN

Perspective: The future of quantum dot photonic integrated circuits

APL Photonics 3, 030901 (2018); https://doi.org/10.1063/1.5021345

Switching between ground and excited states by optical feedback in a quantum dot laser diode

Applied Physics Letters 105, 121109 (2014); https://doi.org/10.1063/1.4896576

Analytical approach to modulation properties of quantum dot lasers

Journal of Applied Physics 109, 103112 (2011); https://doi.org/10.1063/1.3587244

田QBLOX

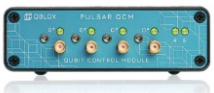

1 qubit
Shorten Setup Time

Auto-Calibration

More Qubits

Fully-integrated

Quantum Control Stacks

Ultrastable DC to $18.5 \mathrm{GHz}$

Synchronized $<<1$ ns

Ultralow noise

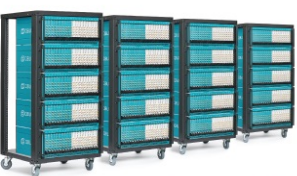

100s qubits

visit our website > 


\title{
Influencing modulation properties of quantum-dot semiconductor lasers by carrier lifetime engineering
}

\author{
Benjamin Lingnau, ${ }^{1}$ Kathy Lüdge, ${ }^{1}$ Weng W. Chow, ${ }^{2}$ and Eckehard Schöll ${ }^{1}$ \\ ${ }^{1}$ Institut für Theoretische Physik, Technische Universität Berlin, 10623 Berlin, Germany \\ ${ }^{2}$ Sandia National Laboratories, Albuquerque, New Mexico 87185-1086, USA
}

(Received 14 February 2012; accepted 10 September 2012; published online 25 September 2012)

\begin{abstract}
The relaxation oscillation (RO) parameters and modulation properties of quantum-dot lasers are investigated depending on effective charge carrier scattering lifetimes of the confined quantum-dot states. We find three dynamical regimes of the laser, characterized by the level of synchronization between carrier dynamics in quantum-dots and quantum-well. For scattering rates similar to the RO frequency, a strong damping is found. On either side of this regime, simulations show low RO damping and improved dynamical response. Depending on the regime, the modulation response differs from conventional analytical predictions. Our results suggest the possibility of tailoring quantum-dot laser dynamical behavior via bandstructure engineering. (C) 2012 American Institute of Physics. [http://dx.doi.org/10.1063/1.4754588]
\end{abstract}

Quantum-dot (QD) lasers offer a variety of advantages over conventional quantum-well (QW) or bulk lasers. ${ }^{1-3}$ Their low threshold currents, high temperature stability, and potentially low chirp make them a preferred choice for many applications. In general, QD lasers show strongly damped relaxation oscillations (ROs), ${ }^{4,5}$ which decreases their sensitivity to optical feedback ${ }^{6,7}$ as well as to optical injection. ${ }^{8,9}$ On the other hand, the possibility for fast dynamic response is limited by the strong damping. ${ }^{4,10}$ The comparably low modulation bandwidth of QD lasers is often attributed to the slow charge carrier capture into the QDs, acting as a bottleneck for the laser dynamics. ${ }^{11}$ However, we show that this explanation is oversimplified.

In this letter, we predict three different dynamical regimes of QD laser dynamics, depending on the charge carrier lifetime. We further show that the modulation bandwidth depends nonlinearly on the scattering lifetimes. Understanding these results is crucial for improving the performance of QD laser devices.

We consider a single-mode dot-in-a-well (DWELL) laser, where the active region consists of 15 QW layers. In each layer, a density $N^{Q D}=10^{11} \mathrm{~cm}^{-2}$ of randomly distributed QDs is embedded. The layers are surrounded and separated by bulk semiconductor material. The QD gain spectrum is considered to be inhomogeneously broadened, with the spectral distribution of the transition energy given by a Gaussian distribution with a standard deviation of $\Delta E=25 \mathrm{meV}$ and a central frequency of $\hbar \omega=0.95 \mathrm{eV}$. The QDs are then distributed into different subgroups (index $j$ ) with the transition energy $\hbar \omega^{j}$ (between electron and hole ground state) and $f(j)$ being the probability to find a QD in the $j$ th subgroup.

The QD laser device is modeled using a semiconductor Maxwell-Bloch approach to cover the coherent dynamics of the polarization as well as the dynamics of the charge carrier distribution within the QD-QW system. As opposed to 3-variable rate equation models ${ }^{12}$ (QW and QD charge carrier densities as well as the photon density), or 5-variable rate equations ${ }^{13,14}$ (differentiating between electrons and holes), the model used here accounts for non-equilibrium and temperature effects. We neglect spatial dependencies and propagation effects ${ }^{15}$ to maintain numerical simplicity. For the QD dynamics, we use the following coupled equations for the electric field amplitude $E$, the microscopic polarization of the $j$ th QD subgroup $p^{j}$, and the occupation probability $\rho_{b}^{j}$ in the $j$ th subgroup $(b=e, h$ labeling electrons and holes, respectively):

$$
\begin{gathered}
\frac{\mathrm{d}}{\mathrm{d} t} E=\frac{i \omega}{2 \varepsilon_{0} \varepsilon_{b g}} \frac{2 \Gamma N^{Q D}}{h^{Q W}} \sum_{j} f(j) \mu p^{j}-\kappa E+\left.\frac{\partial}{\partial t} E\right|_{\text {spont }}, \\
\frac{\mathrm{d}}{\mathrm{d} t} p^{j}=-i\left(\omega^{j}-\omega\right) p-i \frac{\mu E}{2 \hbar}\left(\rho_{e}^{j}+\rho_{h}^{j}-1\right)-\frac{1}{T_{2}} p^{j}, \\
\frac{\mathrm{d}}{\mathrm{d} t} \rho_{b}^{j}=\frac{1}{\hbar} \operatorname{Im}\left(p^{j} \mu^{*} E^{*}\right)-W \rho_{e}^{j} \rho_{h}^{j}+\left.\frac{\partial}{\partial t} \rho_{b}^{j}\right|_{c o l} .
\end{gathered}
$$

Here $\varepsilon_{b g}=11.16$ is the background permittivity, $\Gamma=$ 0.2 is the geometric confinement factor given by the overlap ratio of laser beam and active medium volumes, $h^{Q W}=3 \mathrm{~nm}$ is the height of each QW layer, and $\kappa=0.12 \mathrm{ps}^{-1}$ is the cavity loss rate. The transition dipole moment is given by $\mu=$ $0.6 \mathrm{~nm} \cdot e_{0} \cdot T_{2}=100 \mathrm{fs}$ is a phenomenological dephasing time, leading to polarization decay, and $W=0.4 \mathrm{~ns}^{-1}$ is the Einstein coefficient for the spontaneous emission. The spontaneous emission contribution $\left.(\partial / \partial t) E\right|_{\text {spont }}$ to the electric field is modeled via a complex Gaussian white noise source term $\xi(t)$. In Eq. (3), changes of the charge carrier occupation in the QDs due to scattering events are labeled as $\left.(\partial / \partial t) \rho_{b}^{j}\right|_{c o l}$.

Within the Markov approximation, the scattering processes leading to a charge carrier exchange between QD and QW states can be written in terms of effective Boltzmannlike in- and out-scattering rates $S_{b}^{\text {in/out }}$. Considering the case of unavailability of LO phonons matching the energy separation between QD and QW states and high carrier density for lasing, Auger-type carrier-carrier scattering processes dominate the charge carrier exchange. The corresponding scattering rates exhibit a highly nonlinear dependence on the QW areal charge carrier densities $w_{b}=A^{-1} \sum_{\mathbf{k}} \rho_{b}^{2 D}(\mathbf{k})$ ( $A$ is the 
QW area) as well as the temperature of the charge carrier distribution. ${ }^{16}$ The change of the charge carrier occupation probabilities in the QD subgroups can then be written as

$$
\left.\frac{\partial}{\partial t} \rho_{b}^{j}\right|_{c o l}=S_{b}^{i n}\left(w_{e}, w_{h}\right)\left(1-\rho_{b}^{j}\right)-S_{b}^{\text {out }}\left(w_{e}, w_{h}\right) \rho_{b}^{j} .
$$

In order to derive an effective scattering rate from the above expression, the detailed balance between in- and out-scattering rates, ${ }^{13}$ given by $S_{b}^{\text {out }}=S_{b}^{\text {in }} \exp \left[ \pm\left(\varepsilon_{b}^{Q D}-\right.\right.$ $\left.\left.\mu_{b}^{Q W}\right) /\left(k_{B} T_{b}^{Q W}\right)\right]$ for electrons (+) and holes (-), with the QD ground state (GS) energy $\varepsilon_{b}^{Q D}$, can be utilized. Here, $\mu_{b}^{Q W}$ and $T_{b}^{Q W}$ are the quasi-Fermi level and temperature of the QW charge carrier distribution, respectively. Equation (4) can then be rewritten as

$$
\left.\frac{\partial}{\partial t} \rho_{b}^{j}\right|_{c o l}=\tau_{b}^{-1}\left[f\left(\varepsilon_{b}^{Q D}, \mu_{b}^{Q W}, T_{b}^{Q W}\right)-\rho_{b}^{j}\right]
$$

where the effective (nonconstant) scattering lifetime of the QW and QD charge carrier distribution is given by $\tau_{b} \equiv\left(S_{b}^{i n}+S_{b}^{\text {out }}\right)^{-1}$, defining the rate at which the charge carrier distribution is driven towards the quasi-Fermi distribution $f\left(\varepsilon_{b}^{Q D}, \mu_{b}^{Q W}, T_{b}^{Q W}\right)$.

We proceed within the relaxation rate approximation by replacing $\mu_{b}^{Q W}$ and $T_{b}^{Q W}$ with the quasi-Fermi level and temperature of the quasi-equilibrium distribution of the combined QD-QW system, determined dynamically by charge carrier number and energy conservation conditions. ${ }^{11}$ Since the main message of this letter is to point out the different dynamic regimes of the QD laser dynamics which occur for different values of $\tau_{e}$ and $\tau_{h}$, the $w_{b}$ dependence of these quantities is neglected in the following. Instead, results for different constant lifetimes are shown. When discussing the laser dynamics at a fixed operation point, as done here, the assumption of constant (not $w_{b}$-dependent) scattering times is a good approximation ${ }^{17}$ as long as the individual in- and out-scattering rates fulfill the detailed balance relation. Note, however, that for large changes in the operation parameters (e.g., large-signal modulation), the dependence of $\tau_{b}$ on $w_{b}$ must be taken into account.

The dynamic equations for the $k$-dependent $\mathrm{QW}$ and bulk occupation probabilities $\rho_{b, k}^{2 D}$ and $\rho_{b, k}^{3 D}$, respectively, are given as

$$
\begin{gathered}
\frac{\mathrm{d}}{\mathrm{d} t} \rho_{b, k}^{2 D}=-B_{b}^{S} \rho_{b, k}^{2 D}+\left.\frac{\partial}{\partial t} \rho_{b, k}^{2 D}\right|_{c o l}, \\
\frac{\mathrm{d}}{\mathrm{d} t} \rho_{b, k}^{3 D}=J_{b, k}-B_{b}^{S, b u l k} \rho_{b, k}^{3 D}+\left.\frac{\partial}{\partial t} \rho_{b, k}^{3 D}\right|_{c o l} .
\end{gathered}
$$

Here, $B_{b}^{S}=B^{S} w_{h}\left(B^{S} w_{e}\right)$ for $b=e(h)$ describes bimolecular recombination in the QW, with $B^{S}=540 \mathrm{~ns}^{-1} \mathrm{~nm}^{-2}$, and $B_{b}^{S, b u l k}$ is defined analogously. The electrical pumping is described by $J_{b, k}:=\gamma_{p} f\left(\varepsilon_{b, k}^{3 D}, \mu_{p, b}, T_{\ell}\right)\left(1-\rho_{b, k}^{3 D}\right)$, with a pump rate $\gamma_{p}=1 \mathrm{~ns}^{-1}$ and the bulk state energy $\varepsilon_{b, k}^{3 D}$. The distribution of the injected charge carriers is assumed as a quasiFermi distribution thermalized with the lattice temperature $T_{\ell}$. Its quasi-Fermi level $\mu_{p, b}$ is calculated such that the injected charge carrier density matches the chosen pump current density $J: e_{0} \sum_{\mathbf{k}} J_{b, k} \stackrel{!}{=} A J$. Scattering contributions $\left.(\partial / \partial t) \rho_{k}\right|_{c o l}$ similar to Eq. (5) for each $k$-state have been implemented. Apart from pure carrier-carrier (subscript c-c) scattering events, carrier-phonon (c-p) interaction in the QW and bulk is treated in the same manner, with the corresponding quasi-equilibrium distribution temperature given by the lattice temperature. We use $\gamma_{\mathrm{c}-\mathrm{c}}^{(2)}=2 \mathrm{ps}^{-1}, \gamma_{\mathrm{c}-\mathrm{p}}^{(2)}=0.4 \mathrm{ps}^{-1}$ for the electron scattering rates between QW and bulk (hole rates are twice as fast).

Depending on the QD size and material composition of the heterostructure, the charge carrier scattering rates between QD and QW states can vary over a large range of values. Furthermore, the scattering rates of electrons and holes can differ by more than an order of magnitude. For strongly confined QDs (confinement energies of $\Delta E_{e}$ $=210 \mathrm{meV}, \Delta E_{h}=50 \mathrm{meV}$ for electrons and holes, respectively), our microscopic calculations ${ }^{4}$ reveal a substantially faster scattering of the holes (on average 14 times faster than for electrons), while for a weaker confinement $\left(\Delta E_{e}\right.$ $=74 \mathrm{meV}, \Delta E_{h}=40 \mathrm{meV}$ ), we found that hole rates are similar to the corresponding electron rates, on average 1.1 times as fast. ${ }^{18}$ Both cases of laser devices will be discussed in the following.

First, we look at a device with similar scattering lifetimes $\tau_{b}$ between QW and QD states for electrons and holes and thus assume $\tau_{h}{ }^{-1}=1.1 \tau_{e}^{-1}$. To further discuss the impact of the effective charge carrier scattering rate $\tau_{b}{ }^{-1}$ between QD and QW, the turn-on dynamics is simulated for three different values of $\tau_{b}{ }^{-1}$, which were kept constant throughout each simulation (see Fig. 1(a)). To counteract the lower external quantum efficiency of the device with slower scattering, the pump current was adjusted to yield $I=0.5 \mathrm{MW} \mathrm{cm}^{-2}$ in the steady-state for each simulation.
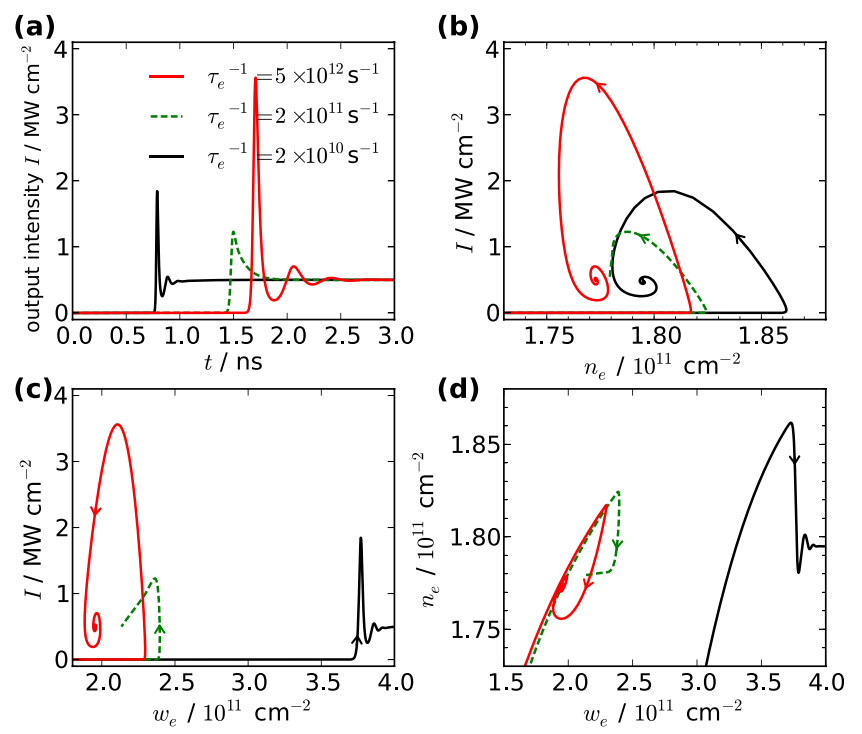

FIG. 1. Turn-on dynamics of the QD laser device with different QW-QD electron effective scattering rates $\tau_{e}^{-1}=2 \times 10^{10} \mathrm{~s}^{-1}$ (solid black line, $\left.J=6.6 j_{t h}\right), \tau_{e}^{-1}=2 \times 10^{11} \mathrm{~s}^{-1}$ (dashed green line, $J=1.8 j_{t h}$ ) and $\tau_{e}^{-1}=5$ $\times 10^{12} \mathrm{~s}^{-1}$ (solid red line, $J=1.5 j_{t h}$ ). $\tau_{h}^{-1}=1.1 \tau_{e}{ }^{-1}$ in all simulations. The injection current was turned on at $t=0$ and chosen to yield the optical intensity $I=0.5 \mathrm{MW} \mathrm{cm}^{-2}$ in the steady-state in all three cases. The turn-on dynamics is shown as (a) time-series of $I=\left(c_{0} \epsilon_{0} \sqrt{\epsilon_{b g}}\right)|E|^{2}$, (b) phase plot of the intensity vs. QD electron density $n_{e}$, (c) phase plot of the intensity vs. QW electron density $w_{e}$, and (d) phase plot of the QD electron density $n_{e}$ vs. QW electron density $w_{e}$. 
Note that the qualitative dependence of the laser dynamics on the scattering rates is independent of the exact value of the chosen intensity. The dynamical change of the bandstructure due to Coulomb energy renormalizations during the turn-on of the laser was previously found to influence the relaxation oscillation behavior of the laser. ${ }^{19}$ Here, in order to isolate the influence of the scattering rate, Coulomb shifts are neglected.

The curves in Fig. 1(a) show that for the slow scattering rate $\tau_{e}^{-1}=2 \times 10^{10} \mathrm{~s}^{-1}$ (black solid curve), the laser intensity $I$ exhibits damped ROs after the initial intensity spike. With increasingly faster scattering rate of $\tau_{e}^{-1}=2$ $\times 10^{11} \mathrm{~s}^{-1}$, the ROs disappear and the laser exhibits overdamped behavior with an initial intensity peak and subsequent exponential decrease of the intensity to its steady-state value (green dashed line). This is in agreement with the analytic result from a rate equation model, where an increase in damping is predicted for increasing inter QD/QW scattering rate. ${ }^{17}$ What is new is that our model predicts the reappearance of ROs when increasing the scattering rates further from this point, as shown by the red solid curve Fig. 1(a), for $\tau_{e}^{-1}=5 \times 10^{12} \mathrm{~s}^{-1}$.

This counterintuitive result can be understood by looking at the phase portraits of the turn-on dynamics shown in Figs. 1(b)-1(d), revealing three different dynamic regimes appearing for the three chosen values of scattering rates. In the $\left(n_{e}, I\right)$ phase space (where $n_{b}=2 N^{Q D} \sum_{j} f(j) \rho_{b}^{j}$ is the areal QD carrier density, with the factor of two accounting for spin degeneracy), the trajectories reach the fixed point (a focus) in a spiraling motion, while the fixed point becomes a node in the strongly damped regime. Note that, due to the similar electron and hole dynamics, the trajectories for the holes yield qualitatively similar results. In the first regime (constant reservoir regime, $\tau_{b}^{-1} \ll \omega_{R O}$ ), corresponding to slow scattering (solid black curve), the amount of charge carriers scattering from the QW states into the QDs during the turn-on is low enough to not influence $w_{e}$ appreciably. After the initial intensity spike, $w_{e}$ and $w_{h}$ thus act as an approximately constant charge carrier reservoir, allowing to eliminate this degree of freedom. ${ }^{17}$ Second, strongly damped regime appears for intermediate scattering rates $\tau_{b}^{-1}=O\left(\omega_{R O}\right) \quad$ (green dashed curve), where the faster charge carrier exchange rate leads to increasingly larger variations of the QW charge carrier density during the turnon. For sufficiently fast scattering (solid red curve), the third dynamic regime (synchronized regime, $\tau_{b}^{-1} \gg \omega_{R O}$ ) is reached, where the charge carrier exchange is fast enough for the QW and QD charge carrier distributions to be close to quasi-equilibrium at all times. The QW densities then adiabatically follow the QD density variations during the turn-on, which can be seen in Fig. 1(c), where the trajectory in the $\left(w_{e}, I\right)$ phase space shows a spiral similar to that in the $\left(n_{e}, I\right)$ phase space. The dynamic degree of freedom of the QW equations can thus be adiabatically eliminated and the system behavior approaches that of a QW laser, ${ }^{20}$ with the QD and QW acting as a combined charge carrier system.

We now take a closer look at the dynamics for the second case of a device with different electron and hole lifetimes where the hole scattering is in average 14 times as fast as the corresponding electron rate. The dependence of the relaxation oscillation damping rate $\Gamma_{R O}$ (blue circles) and frequency $f_{R O}$ (red squares) on the electron scattering rate is shown in Fig. 2(a). The RO parameters were extracted from the intensity time series by fitting the intensity time-series with a damped sinusoidal oscillation. Additionally, the results for the device with similar scattering lifetimes of electrons and holes discussed before are plotted in Fig. 2(b). For both cases, the damping rate $\Gamma_{R O}$ is largest in the 2 nd regime at intermediate values of the scattering rates, where these are comparable to $\omega_{R O}=2 \pi f_{R O}$, as seen in Fig. 1. For different electron and hole lifetimes, this peak is around $\tau_{e}^{-1}=2 \times 10^{10} \mathrm{ps}^{-1}\left(\tau_{e}^{-1}=2 \times 10^{11} \mathrm{ps}^{-1}\right.$ for similar lifetimes). The damping becomes smaller both when decreasing or increasing the scattering rates from that point. For similar lifetimes, shown in Fig. 2(b), even more pronounced changes in the relaxation oscillation behavior can be observed, i.e., the steep rise and subsequent jump of the RO damping prior to the overdamped region where no ROs can be observed (between the two dashed vertical lines).

From Fig. 2, it becomes evident that assuming different electron and hole dynamics (different $\tau_{e}$ and $\tau_{h}$ ) leads to fundamentally different dynamic scenarios and crucially affects the dependence of the RO parameters on QD scattering lifetimes. Thus adjusting the device material structure allows one to drastically change the laser dynamics.

The modulation properties of the laser device are often determined from the RO parameters by assuming an analytical expression for the modulation response of the laser device $^{21}$

$$
H(\omega)=\frac{\omega_{0}^{4}}{\left(\omega_{0}^{2}-\omega^{2}\right)^{2}+4 \Gamma_{R O}^{2} \omega^{2}},
$$
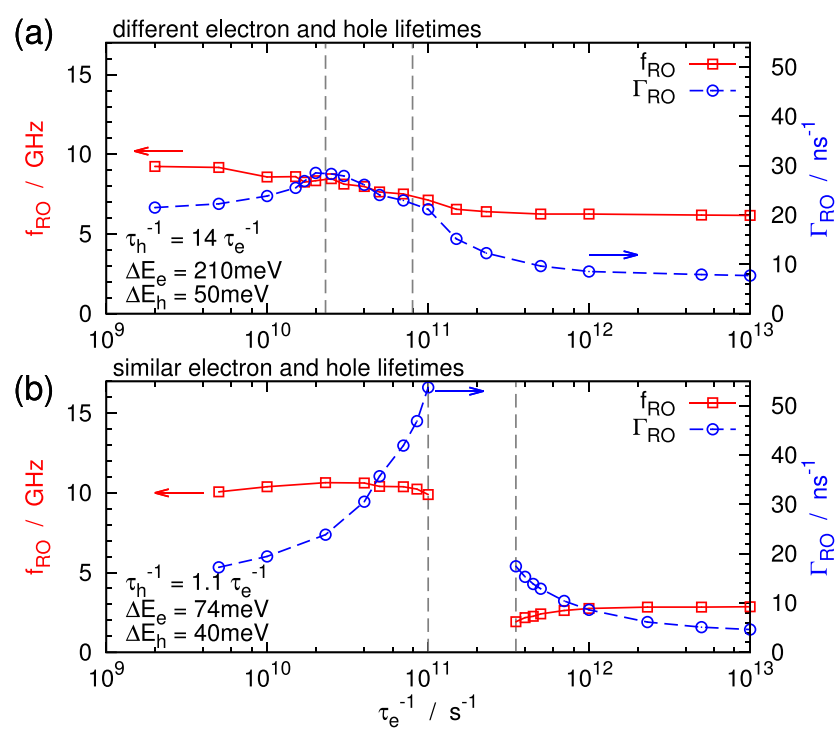

FIG. 2. RO frequency $f_{R O}$ (red squares) and damping coefficient $\Gamma_{R O}$ (blue circles) dependences on $\tau_{e}{ }^{-1}$. The pump current was chosen to yield fixed optical output $I=0.5 \mathrm{MW} \mathrm{cm}^{-2}$ at steady state for each data point. (a) Different electron and hole lifetimes: $\tau_{h}{ }^{-1}=14 \tau_{e}{ }^{-1}$. (b) Similar electron and hole lifetimes: $\tau_{h}{ }^{-1}=1.1 \tau_{e}^{-1}$. The dashed gray vertical lines delimit the three different dynamic regimes (from left to right: constant reservoir regime, strongly damped regime, and synchronized regime). 


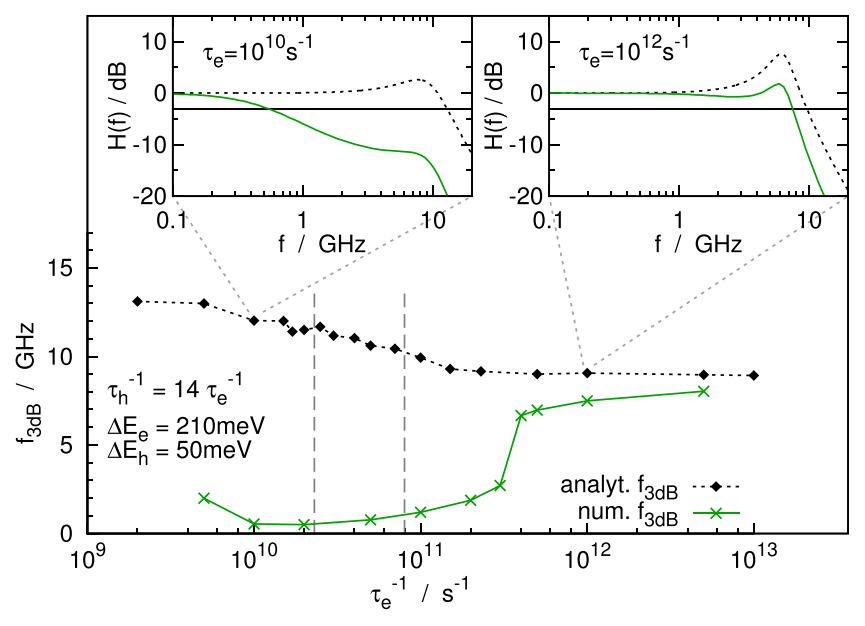

FIG. 3. Analytical (dashed black curve) and numerically obtained modulation response (solid green curve) for different electron scattering lifetimes $\left(\tau_{h}^{-1}=14 \tau_{e}^{-1}\right)$, with the injection current chosen to yield $I=0.5 \mathrm{MWcm}^{-2}$ at steady state for each data point. The insets show the numerical (green solid lines) and analytical (black dashed lines) modulation response curves at $\tau_{e}^{-1}=10^{10} \mathrm{~s}^{-1}$ and $10^{12} \mathrm{~s}^{-1}$, respectively, with the horizontal line showing the $-3 \mathrm{~dB}$ level.

with $\omega_{0}^{2} \approx \omega_{R O}^{2}+\Gamma_{R O}^{2}$. Solving $H\left(\omega_{3 \mathrm{~dB}}\right) \stackrel{!}{=} \frac{1}{2}$ yields the $3 \mathrm{~dB}$ bandwidth $f_{3 d B}$ as a function of $\Gamma_{R O}$ and $f_{R O}$ and thus of $\tau_{e}{ }^{-1}$. The $3 \mathrm{~dB}$-bandwidth predicted from the RO parameters is shown in Fig. 3 (dashed black line), along with the numerically determined bandwidth (solid green line).

The analytical expression predicts an increase of the modulation bandwidth with decreasing scattering rates, related to the higher RO frequency observed at low values of $\tau_{e}^{-1}$. The numerically evaluated modulation response, however, reveals a sharp decrease of the modulation bandwidth below $\tau_{e}^{-1} \approx 5 \times 10^{11} \mathrm{~s}^{-1}$.

Especially in the vicinity of the high damping regime at intermediate scattering rates, the modulation bandwidth is thus reduced to values below $1 \mathrm{GHz}$, as opposed to the bandwidth of $>10 \mathrm{GHz}$ predicted from the RO parameters via Eq. (8). The reason for this discrepancy can be seen in the insets in Fig. 3, where the modulation response of the QD laser for $\tau_{e}^{-1}=10^{10} \mathrm{~s}^{-1}$ and $\tau_{e}^{-1}=10^{12} \mathrm{~s}^{-1}$ is shown. For $\tau_{e}^{-1}$ $=10^{10} \mathrm{~s}^{-1}$, the modulation response curves reveal a decline at low modulation frequencies, while they approach Eq. (8) for increasing scattering rates. These differences can be attributed to the inter-QD/QW scattering processes not accounted for in Eq. (8). ${ }^{22}$ The analytical expression describes the numerical modulation response at fast scattering rates very well in the case of similar electron and hole lifetimes, where the QD and QW distributions are strongly coupled. For different carrier lifetimes, however, some differences remain even at high $\tau_{e}^{-1}$, as seen in Fig. 3, revealing that the modulation response is negatively influenced by the different electron and hole dynamics.

In conclusion, we have shown that in QD lasers, there exist three different dynamical regimes, depending on the scattering lifetime of charge carriers between QW and QD states in relation to the RO frequency. Both for very low (constant reservoir regime) and very high scattering rates (synchronized regime), the QW dynamical variables can be eliminated and pronounced relaxation oscillations are observed. For intermediate scattering rates in the order of the RO angular frequency (strongly damped regime), strongly damped relaxation oscillations are found, which limits laser dynamical performance, as is observed in experiments. The RO frequency is found to be highest for scattering rates in the constant reservoir regime. Taking these dynamical regimes into account allows one to improve the dynamic response of QD lasers by carefully adjusting the device optical and material structures.

This work was supported by DFG within Sfb 787, the U.S. Department of Energy's National Nuclear Security Administration under Contract DE-ACD4-94AL85000, and Sandia's Solid-State Lighting Science Center, an Energy Frontier Research Center (EFRC) funded by the U.S. Department of Energy, Office of Science, Office of Basic Energy Sciences.

${ }^{1}$ D. Bimberg, M. Grundmann, and N. N. Ledentsov, Quantum Dot Heterostructures (John Wiley \& Sons Ltd., New York, 1999).

${ }^{2}$ D. Bimberg, Semiconductor Nanostructures, edited by D. Bimberg (Springer, Berlin, 2008).

${ }^{3}$ K. Lüdge, Nonlinear Laser Dynamics - From Quantum Dots to Cryptography, edited by K. Lüdge (Wiley-VCH, Weinheim, 2012).

${ }^{4}$ K. Lüdge, R. Aust, G. Fiol, M. Stubenrauch, D. Arsenijević, D. Bimberg, and E. Schöll, IEEE J. Quantum Electron. 46, 1755 (2010).

${ }^{5}$ M. Kuntz, N. N. Ledentsov, D. Bimberg, A. R. Kovsh, V. M. Ustinov, A. E. Zhukov, and Y. M. Shernyakov, Appl. Phys. Lett. 81, 3846 (2002).

${ }^{6}$ C. Otto, K. Lüdge, and E. Schöll, Phys. Status Soldi B 247, 829 (2010).

${ }^{7}$ C. Otto, B. Globisch, K. Lüdge, E. Schöll, and T. Erneux, "Complex Dynamics of Semiconductor Quantum Dot Lasers Subject to Delayed Optical Feedback," Int. J. Bifurcation Chaos Appl. Sci. Eng. (in press).

${ }^{8}$ J. Pausch, C. Otto, E. Tylaite, N. Majer, E. Schöll, and K. Lüdge, New J. Phys. 14, 053018 (2012).

${ }^{9}$ B. Kelleher, C. Bonatto, G. Huyet, and S. P. Hegarty, Phys. Rev. E 83, 026207 (2011).

${ }^{10}$ M. Ishida, M. Sugawara, T. Yamamoto, N. Hatori, H. Ebe, Y. Nakata, and Y. Arakawa, J. Appl. Phys. 101, 013108 (2007).

${ }^{11}$ W. W. Chow and S. W. Koch, IEEE J. Quantum Electron. 41, 495 (2005).

${ }^{12}$ D. O'Brien, S. P. Hegarty, G. Huyet, and A. V. Uskov, Opt. Lett. 29, 1072 (2004).

${ }^{13}$ K. Lüdge and E. Schöll, IEEE J. Quantum Electron. 45, 1396 (2009).

${ }^{14} \mathrm{M}$. Gioannini and I. Montrosset, IEEE J. Quantum Electron. 43, 941 (2007).

${ }^{15}$ M. Gioannini and M. Rossetti, IEEE J. Sel. Top. Quantum Electron. 17, 1318 (2011).

${ }^{16}$ N. Majer, K. Lüdge, and E. Schöll, Phys. Rev. B 82, 235301 (2010).

${ }^{17}$ K. Lüdge, E. Schöll, E. A. Viktorov, and T. Erneux, J. Appl. Phys. 109, 103112 (2011).

${ }^{18}$ N. Majer, S. Dommers-Völkel, J. Gomis-Bresco, U. Woggon, K. Lüdge, and E. Schöll, Appl. Phys. Lett. 99, 131102 (2011).

${ }^{19}$ B. Lingnau, K. Lüdge, E. Schöll, and W. W. Chow, Appl. Phys. Lett. 97, 111102 (2010).

${ }^{20}$ T. Erneux, E. A. Viktorov, and P. Mandel, Phys. Rev. A 76, 023819 (2007).

${ }^{21}$ M. Sugawara, N. Hatori, M. Ishida, H. Ebe, Y. Arakawa, T. Akiyama, K. Otsubo, T. Yamamoto, and Y. Nakata, J. Phys. D 38, 2126 (2005).

${ }^{22}$ C. Wang, F. Grillot, and J. Even, IEEE J. Quantum Electron. 48(9), 1144 (2012). 